\title{
Tracking the state transitions in changing-look active galactic nuclei through their polarized-light echoes
}

\author{
F. Marin ${ }^{1}$ and D. Hutsemékers ${ }^{2}$ \\ ${ }^{1}$ Université de Strasbourg, CNRS, Observatoire Astronomique de Strasbourg, UMR 7550, 67000 Strasbourg, France \\ e-mail: frederic.marin@astro. unistra.fr \\ 2 F.R.S.-FNRS, Institut d'Astrophysique et de Géophysique, Université de Liège, Allée du 6 Août 19c, 4000 Liège, Belgium \\ Received 18 June 2019 / Accepted 12 March 2020
}

\begin{abstract}
Context. Variations in the mass accretion rate appear to be responsible for the rapid transitions in spectral type that are observed in increasingly more active galactic nuclei (AGNs). These objects are now labeled "changing-look" AGNs and are key objects for understanding the physics of accretion onto supermassive black holes.

Aims. We aim to complement the analysis and interpretation of changing-look AGNs by modeling the polarization variations that can be observed, in particular, polarized-light echoes.

Methods. We built a complex and representative model of an AGN and its host galaxy and ran radiative transfer simulations to obtain realistic time-dependent polarization signatures of changing-look objects. Based on actual data, we allowed the system to become several times fainter or brighter within a few years, assuming a rapid change in accretion rate.

Results. We obtain time-dependent polarization signatures of distant high-luminosity (quasars) and nearby low-luminosity (Seyferts) changing-look AGNs for a representative set of inclinations. We predict the evolution of the continuum polarization for future polarimetric campaigns with the goal to better understand the physics at work in these objects. We also investigate highly inclined AGNs that experience strong accretion rate variations without appearing to change state. We apply our modeling to Mrk 1018, the best-documented case of a changing-look AGN, and predict a variation in its polarization after the recent dimming of its continuum. Conclusions. We demonstrate that polarization monitoring campaigns that cover the transitions that are observed in changing-look AGNs might bring crucial information on the geometry and composition of all the reprocessing regions within the nucleus. In particular, specific features in the time variation of the polarization position angle can provide a new and efficient method for determining AGN inclinations.
\end{abstract}

Key words. galaxies: active - galaxies: Seyfert - polarization - quasars: general - radiative transfer - scattering

\section{Introduction}

The optical spectrum of type 1 active galactic nuclei (AGNs) is characterized by broad and narrow emission lines, while the spectrum of type 2 AGNs only show narrow emission lines. Faint broad $\mathrm{H} \alpha$ emission remains detectable in type 1.9 AGN spectra, yet a faint broad $\mathrm{H} \alpha$ emission line can alternatively mean that some scattered light is detected in the total flux, as in the case of 3C 234 (Antonucci 1984). Changinglook AGNs (CLAGNs) constitute a rare class of AGNs that change their spectral type from type 1 to type $1.9 / 2$ or vice versa on timescales that can be shorter than a few years. CLAGNs with appearing or disappearing broad emission lines (BELs) were first discovered among Seyfert galaxies, that is, nearby and low-luminosity AGNs (e.g., Khachikian \& Weedman 1971; Cohen et al. 1986; Goodrich 1989). Recently, appeareance and disappeareance of BELs was also discovered in highluminosity AGNs (quasars; LaMassa et al. 2015; Runnoe et al. 2016; Ruan et al. 2016; MacLeod et al. 2016). These spectral changes are accompanied by a dimming or a brightening of the continuum; the dimming corresponds to changes from type 1 to type 1.9/2, and the brighten to changes from type 1.9/2 to type 1. New CLAGNs are now regularly identified (e.g., Gezari et al. 2017; Assef et al. 2018; Stern et al. 2018; Wang et al. 2018; Yang et al. 2018; MacLeod et al. 2019).
The observed changes might be caused by modifications in the source of ionizing radiation, likely a variation in the rate of accretion onto the supermassive black hole (SMBH): an intrinsic dimming of the continuum source reduces the number of photons available to ionize the gas, resulting in a net decrease of the emission line intensity (e.g., Penston \& Perez 1984; Elitzur et al. 2014; Noda \& Done 2018). Alternatively, variable dust absorption due to the motion of individual clouds in a clumpy torus might occult the continuum source and the broad line region (e.g., Goodrich 1989; Tran et al. 1992). The variable dust absorption scenario is disfavored in changinglook quasars because the timescales of extinction variations due to dusty clouds moving in front of the broad line region are factors 2-10 too long to explain the observed spectral changes (LaMassa et al. 2015; MacLeod et al. 2016). In addition, MacLeod et al. 2016 have shown that if extinction by dust can easily explain the great change in the continuum flux and $\mathrm{Mg}$ II line in several of their CLAGNS, variable extinction cannot explain the observed change in $\mathrm{H} \beta$. Sheng et al. (2017) found large variations of the mid-infrared luminosity that echoed the optical variations that occur during the change of look. The authors argued that this behavior is inconsistent with the variable obscuration scenario because of the excessive amount of extinction needed and the too long obscuration timescale. Finally, the small continuum polarization degree measured in 
most changing-look quasars also argues against variable obscuration (Hutsemékers et al. 2017, 2019). If the disappearance of BELs in changing-look quasars is indeed caused by clouds hiding the quasar core, only light scattered by polar material (if any; see, e.g., Reeves et al. 2016) can reach the observer. The quasar light is thus expected to be highly polarized when the nucleus is seen at not-too-low inclinations. While for nearly all changing-look quasars the observed changes are better attributed to a variation in the SMBH accretion rate, by contrast, changes of look observed in Seyferts are explained by either variation in the accretion rate (e.g., Mrk 1018, Husemann et al. 2016; Mrk 590, Denney et al. 2014; NGC 2617, Shappee et al. 2014) or variable obscuration (e.g., NGC 7603, NGC 2622, Goodrich 1989; Mrk 993, Tran et al. 1992). This distinction is not definitive, however, because objects where variable obscuration is thought to be responsible for the change of look often show complex signatures that cannot always be explained by a change in extinction alone. For example, in the case of NGC 3516, correlated changes in the optical continuum and BELs, strong absorption in the UV lines, as well as in the X-ray continuum can be explained with variable obscuration (Shapovalova \& Popović 2019). However, shoulder-like structures in the wings of the broad $\mathrm{H} \alpha$ and $\mathrm{H} \beta$ emission line profiles seem to indicate that the structure of the BEL region itself has significantly changed during the CLAGN activity minimum (Shapovalova \& Popović 2019).

In the framework of the intrinsic variability scenario, we expect a time delay between the dimming of the continuum seen in direct light and the dimming of the scattered continuum seen in polarized light, in particular for CLAGNs seen at intermediate to high inclinations (Hutsemékers et al. 2017, 2019). The light scattered in polar regions that extend over tens to hundreds of parsecs (Capetti et al. 1995; Kishimoto et al. 2002; Zakamska et al. 2005) can reach the observer decades to centuries after the direct light in the most extreme cases. Fortunately, light echoes most likely originate from the base of the wind (Marin et al. 2012), where shorter (months to years) time delays are expected. In this case, the polarized light contains the echo of a past bright phase diluted by the much fainter direct light, then resulting in a high polarization degree that is expected to slowly decrease with time. Hutsemékers et al. (2019) showed that such a scenario is supported by time-dependent radiative transfer simulations, and argued that it can explain the high polarization measured in the changing-look quasar J022652.24-003916.5. BELs are also expected to be observed in the polarized light, constituting echoes of a past type 1 phase.

In the present paper, we further explore the effect of an intrinsically variable continuum source on AGN polarization. Based on time-dependent radiative transfer simulations, we produce polarization time series for various realistic CLAGN models. In Sect. 2 we describe the radiative transfer code and the generic AGN model we use to obtain time-dependent polarization signatures of CLAGNs. We investigate both dimming and brightening scenarios for quasars and Seyfert-like objects seen at various inclinations. Using the best-documented observational case, we apply our simulations to Mrk 1018 in Sect. 3 to show how the polarization signatures are expected to vary within the next decades. We discuss the constraints that future polarimetric monitoring campaigns of known CLAGNs might advance our understanding of the morphology and composition of AGNs, and examine the most promising cases in Sect. 4. We finally conclude our analysis in Sect. 5 by summarizing the benefits of regular polarimetric observations of AGNs and the importance of spectropolarimetric observations in addition to single broadband-imaging polarimetric measurements.

\section{Modeling polarized echoes}

We start our investigation by constructing a generic AGN model in Sect. 2.1. We explore its polarization properties from a static point of view in Sect. 2.2, that is, when the AGN does not experience a strong change in its optical classification. We then allow the model to evolve by dimming or brightening its nuclear flux by a factor 10 in 6 years in Sect. 2.3. This corresponds to the timescale of the observed BEL disappearance in the quasar J022652.24-003916.5 (MacLeod et al. 2016; Hutsemékers et al. 2019).

\subsection{AGN model}

We built a radio-quiet AGN prototype on the basis of the unified model (Antonucci 1993). At the center of the simulation lies an SMBH and its accreting and emitting engine, which we modeled using a point-like source that emits unpolarized photons isotropically. In the remainder of this article, we focus on mono-energetic analyses at UV-visible wavelengths, therefore the exact spectral energy distribution does not matter. Around the central engine, we added an equatorial, electron-filled, uniform flared-disk component that is necessary to explain the temporal variations in the polarization degree of the $\mathrm{H} \alpha$ and continuum emission, as well as the polarization position angle of the broad $\mathrm{H} \alpha$ wings, as observed in nearby AGNs (Young 2000). Because the variations observed in the polarized flux are slower than the observed variations in the direct view of the broad lines (Young et al. 1999), this scattering region must be larger than the BEL and extend up to the torus. The half-opening angle of the flared disk was set to $20^{\circ}$ from the equatorial plane and its optical thickness in the $V$-band was set to $\tau_{V}=1$. Both values were derived from the extensive grid of models presented in Marin et al. (2012). They allow us to reproduce the expected optical polarization signatures of nearby Seyfert galaxies. The inner and outer radii of this scattering region were set to 0.001 and $0.1 \mathrm{pc}$, respectively. The inner radius corresponds to a distance large enough so that the central engine can be approximated by a point source, in agreement with self-consistent dynamical models of the BEL region (Czerny et al. 2017). The outer radius was bounded by the dust sublimation radius that defines the beginning of the circumnuclear region, which is usually referred to as the torus. Its inner radius is luminosity dependent and scales as $R_{\text {in }} \approx 0.4 L_{45}^{1 / 2} T_{1500}^{2.6}$ pc (Nenkova et al. 2008a,b). For a fiducial dust sublimation temperature $T=$ $1500 \mathrm{~K}$ and a bolometric luminosity $L=10^{45} \mathrm{erg} \mathrm{s}^{-1}$, the inner radius of the torus is a fraction of a parsec, under the hypothesis of an optically thick dusty region and for a specific composite dust grain structure (Elitzur 2008). We modeled the torus using a flared-disk region, and we filled it with dust grains $(37.5 \%$ graphite and $62.5 \%$ astronomical silicate). The grain radii ranged from 0.005 to $0.250 \mu \mathrm{m}$ and the grain size index was set to -3.5. The model was the usual cosmic dust of the Milky Way (Mathis et al. 1977) and the optical thickness of this uniformly filled medium was in excess of 50, so that the torus was optically thick to ultraviolet and optical radiation. The half-opening angle of the torus was set from observational constraints based on quasar populations (Sazonov et al. 2015; Marin 2016) and fixed at $45^{\circ}$. Its outer radius was fixed by mid-infrared interferometric measurements in nearby AGN, together with dust radiative transfer simulations that all point toward a rather compact torus $\left(R_{\text {out }} \sim 5-10 \mathrm{pc}\right)$ embedded in a larger diffuse dusty region (Pier \& Krolik 1992; Meisenheimer et al. 2008). We therefore set this outer radius to $5 \mathrm{pc}$. The dusty structure collimates the 
winds from the central engine. They take the form a biconical structure that extends up to $100 \mathrm{pc}$ along the polar direction, the so-called ionization cones resolved in nearby Seyferts (Antonucci et al. 1994; Simpson et al. 1997). The optical depth of the medium was chosen to be 0.1 so that a non-negligible fraction of the photons can scatter inside the polar medium (Marin et al. 2012). Finally, around the AGN model, we added a geometrically thin disk-like structure representative of the host galaxy. It was assumed to be perpendicular to the AGN symmetry axis and to extend up to several kiloparsecs. This is not the most general case, however, because it is known that the position angles of AGNs and their host are uncorrelated (Ulvestad \& Wilson 1984; Kinney et al. 2000). This choice is due to limitations of our simulation tool. The host galaxy emits unpolarized starlight that dilutes the observed polarization from the central engine. Absorption and/or scattering by dust in the host were not taken into account. By doing so, we mitigated the effect of the aligned AGN/host configuration by preventing scattering-induced or dichroic absorption polarization from the host galaxy (Thompson \& Martin 1988). In addition, because we work under the approximation of single-wavelength photons, reddening of the type 1 spectra by material in the host plane is not a concern either. In our simulations, the amount of starlight is fixed; only the nuclear light from the AGN can dim or brighten due to mass accretion rate variations (Noda \& Done 2018). An unscaled sketch of the AGN model is presented in Fig. 1 to illustrate the complexity of the simulations that coherently couple emitting, absorbing, and reprocessing regions from milliparsec to kiloparsec scales.

\subsection{Static polarization properties}

To simulate the radiative transfer of photons from the SMBH and its accretion disk to the observer, we used the Monte Carlo code STOKES (Goosmann \& Gaskell 2007; Marin et al. 2012, 2015; Rojas Lobos et al. 2018; Marin 2018). The code allows us to reproduce the radiative coupling of photons that travel within a complex three-dimensional environment. All scattering physical processes that are relevant in the optical regime (Thomson, Mie, Rayleigh) are included in the version of the code we used, and were used when the spectropolarimetric signal of CLAGNs was considered. The code is available online and can be used freely ${ }^{1}$. Photons that successfully escaped the model are registered by a virtual web of detectors in all polar and azimuthal angles at once. The Stokes parameters of light allow us to reconstruct the observed polarization degree $P$ and polarization position angle $\Psi$. In the following we denote with $\Psi$ the polarization position angle defined with respect to the AGN symmetry axis, $\Psi=90^{\circ}$ indicating polarization parallel to the axis. We denote with $\theta$ the polarization position angle measured with respect to the equatorial north-south direction, $\theta=90^{\circ}$ corresponding to the eastwest direction. For the remainder of this paper, we concentrate our efforts on the continuum polarization, and we constrain the source to emit at a central wavelength of $2900 \AA$. This value is derived from the recent observations of changing-look quasar polarization by Hutsemékers et al. (2019), who measured the continuum polarization of J022652.24-003916.5 at $2900 \AA$ (in the quasar rest frame). Additionally, in the near-ultraviolet band, the amount of starlight diminishes, which increases our chances of observing the true nuclear polarization signal. We simulated $10^{9}$ photons for each realization and azimuthally integrated the signal due to the axisymmetric nature of the model. With the

\footnotetext{
1 http://stokes-program. info
}

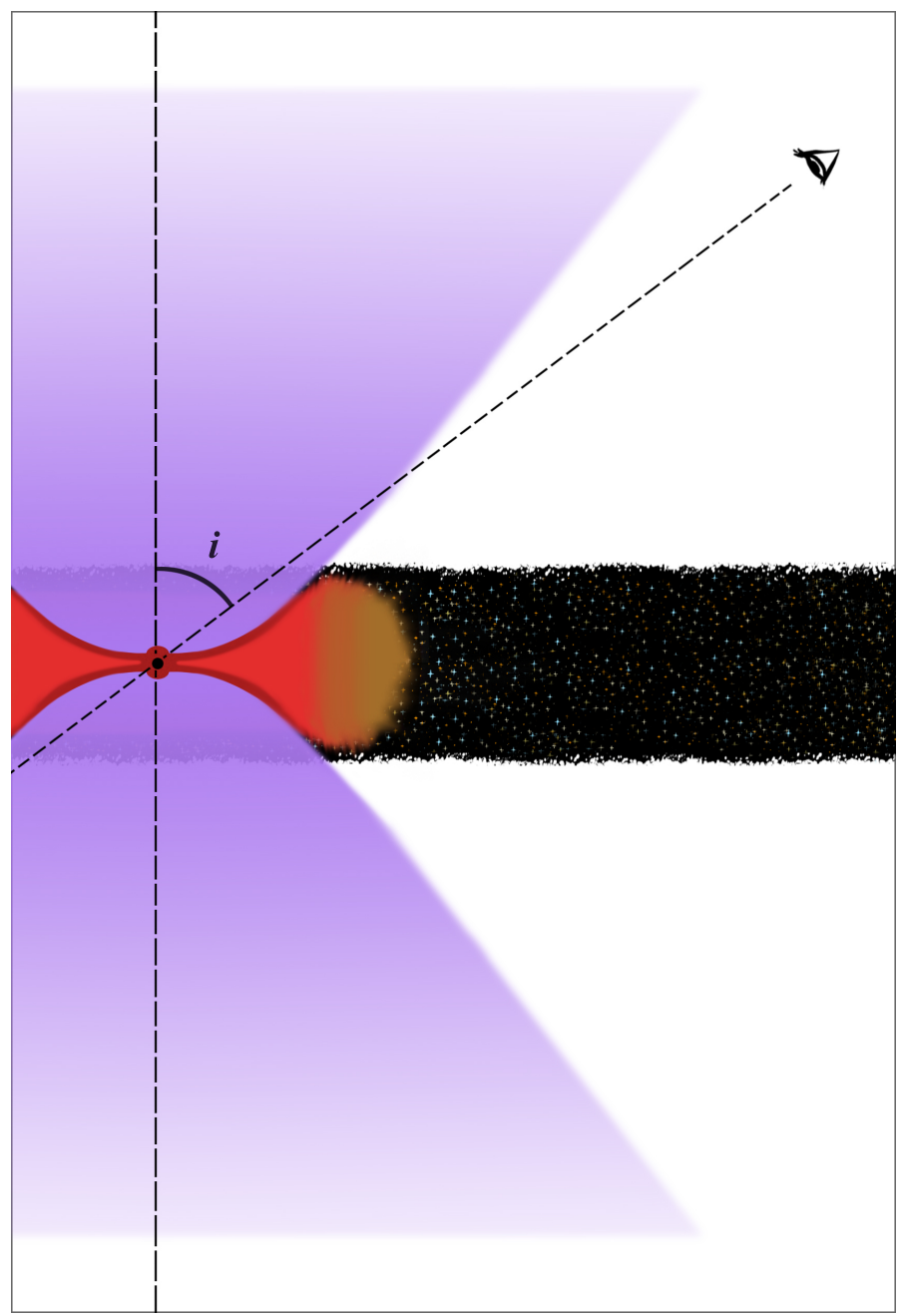

Fig. 1. Unscaled illustration of the AGN model components. The central SMBH and its accretion disk are merged into a punctual source represented with a black dot. By moving away from the SMBH, the source becomes the electron-scattering region (red). The equatorial electron scattering region becomes the circumnuclear dusty torus (brown). The inner dusty funnel collimates disk-born polar winds (violet). Finally, the host galaxy is shown in black. The vertical long-dashed line is the symmetry axis of the AGN. The short-dashed line is the observer's viewing angle, whose inclination is defined with respect to the symmetry axis of the model. See text for further details.

exception of the results presented in Fig. 2 that took only $7 \mathrm{~h}$ to complete, the simulations took $\sim 50 \mathrm{~h}$ each on a regular desktop computer.

In Fig. 2 we explore the inclination-dependent polarization degree (top) and angle (bottom) from our AGN model (solid black line). The contribution of the host galaxy to the total flux was set to 5\% (typical value for quasars; see, e.g., Kauffmann et al. 2003). The polarization degree increases with increasing inclination. This is due to the departure of the centrosymmetric pattern of polarization vectors seen at $i=0^{\circ}$. The polarization degree reaches $1.4 \%$ at most around $i=35^{\circ}$. The associated polarization position angle is $90^{\circ}$, which corresponds to a polarization angle parallel to the symmetry axis of the model according to the convention used in STOKES. This is the expected polarization angle observed in both type 1 quasars and Seyfert galaxies (Antonucci 1993). When the observer's line of sight reaches the geometrical limits of the circumnuclear dusty region, there is a canceling action from parallel $\Psi$ emerging from 

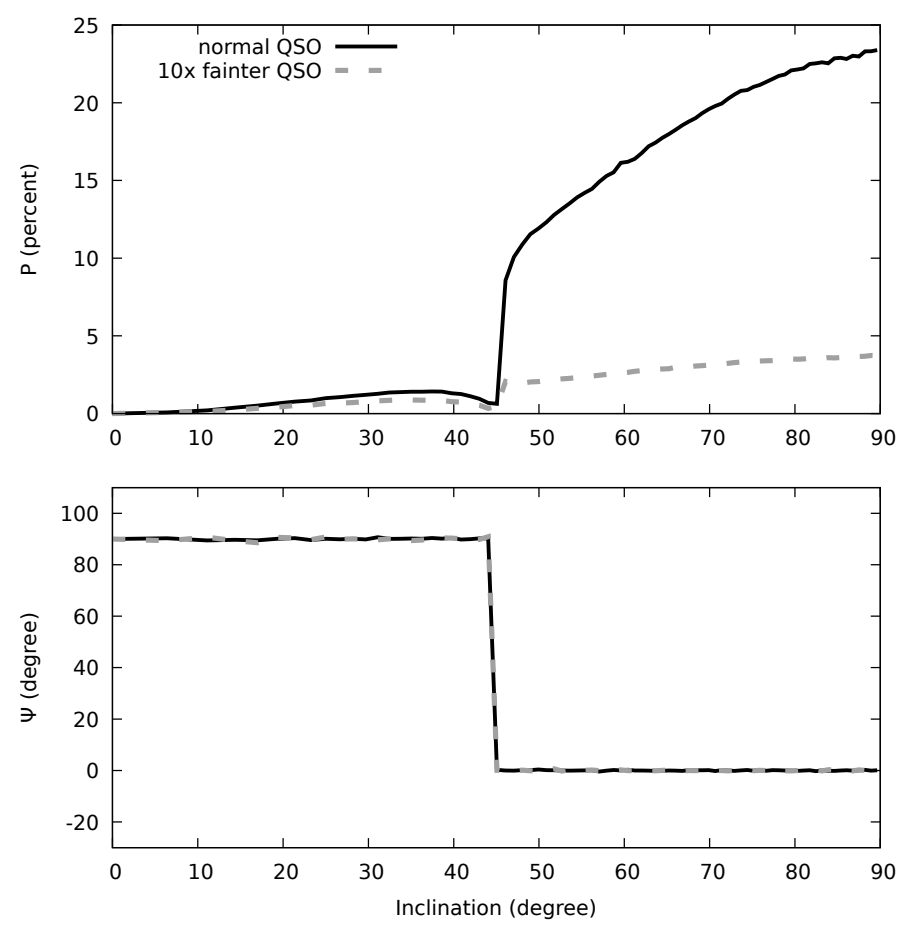

Fig. 2. Polarization degree (top) and polarization position angle (bottom) from our AGN model. The black line corresponds to a bright quasar. In this case, the host galaxy contributes $\sim 5 \%$ of the total light along type 1 inclinations $\left(i \leq 45^{\circ}\right)$. The long-dashed gray line corresponds to the exact same quasar, but ten times fainter. The small fluctuations in $P$ are due to statistical noise.

the equatorial regions and perpendicular $\Psi$ originating from the polar regions. The polarization degree decreases and becomes null around $i=45^{\circ}$. Then $P$ rises again with inclination and $\Psi$ becomes perpendicular $\left(\Psi=0^{\circ}\right)$ because the central engine is now obscured by the optically thick dusty layer and the photons we observe have been scattered inside the polar winds, resulting in higher polarization degrees (up to 23\%). High polarization degrees like this are common after correction for the stellar light (e.g., Kay 1994), and some broad line polarizations or some spatially resolved regions of NGC 1068 have even higher values (Antonucci 2002; Simpson et al. 2002).

We then decreased the contribution from the central engine to the total flux to estimate the polarization we would observe from a quasar that is ten times fainter. This corresponds to the Seyfert galaxy case where the host or AGN flux ratio has increased by a factor 10 . We caution that in this example, the model is static: the flux did not dim from a bright state, and we instead considered the AGN to be naturally less bright. Figure 2 (dashed gray line) shows that a fainter AGN presents a slightly lower polarization degree along type 1 viewing angles (between $0^{\circ}$ and $45^{\circ}$ ). On average, the polarization is 1.5 times lower because the starlight dilution is stronger. Along type 2 inclinations $\left(i=45^{\circ}-90^{\circ}\right)$, the difference is much more notable, with a variation by a factor $\sim 6$ because the central engine is only seen through reprocessed emission that is ultimately heavily diluted by the host emission. We note, however, that the polarization position angle does not change between a quasar and its ten times fainter counterpart.

\subsection{Polarized echoes of CLAGNs}

We now investigate the time-dependent variations in polarization that result from a dimming or brightening of the central
AGN. We use the exact same model investigated in Sect. 2.2 and focus on the most luminous objects (quasars) for observational reasons: their host or AGN flux ratio is much more favorable to the detection of linear continuum polarization in the nearultraviolet and optical wavelength bands. First, we set the quasar to its bright state for 50 years, then allow a linear decrease in quasar flux by a factor 10 in 6 years. We then track the evolution of polarization during the next century in Sect. 2.3.1. Second, in Sect. 2.3.2, we use the faint quasar model and turn on the central engine to determine whether the polarization behavior is symmetric to the dimming case or if it has unique signatures. Finally, we compare quasar and Seyfert changing-look AGNs in Sect. 2.3.3.

\subsubsection{Dimming quasars}

We present the results of our polarimetric investigations of dimming quasars in Figs. 3a-d. The first two panels show the timedependent signal from type 1 quasars, that is, objects seen at an inclination of $10^{\circ}$ and $45^{\circ}$, respectively. In both cases the central engine is directly visible through the equatorial dust funnel, although in the second case the observer's line of sight grazes the dust horizon. The other two panels show the polarization expected from type 2 quasars, that is, when the extinction of the nucleus is total, at inclinations $60^{\circ}$ and $80^{\circ}$, respectively. The panels show the total flux variation (top panel), polarization degree (middle), and polarization position angle (bottom) as a function of time. In the total flux panel, we show the contribution of the quasar, the host galaxy, and the combination of both. We indicate the onset and end of the dimming phase using vertical red lines.

The type 1 objects (Figs. 3a and b) show that the flux variation is rather straightforward. The flux diminishes by a factor close to but not exactly 10 in 6 years (the simulation in Fig. $3 b$ was shown in Hutsemékers et al. 2019, where the quoted fiveyear dimming corresponds to the bulk of the flux drop). This is due to the contribution of the host galaxy, which remains constant with time. During the bright quasar state, the host only accounts for $5 \%$ of the total flux from the system, but when the central engine is in the low state, the relative contribution of the host is higher, almost $30 \%$. After the tenfold decay, the AGN optical type classification can change from type 1 to true type 2 (i.e., intrinsically lacking a BEL emitting region). Note that it is still debated whether true type $2 \mathrm{~s}$, intrinsically lacking broad line emission and in some cases, a thermal big blue bump truly exist (Antonucci 2012). One of the best representative case of true type-2s, NGC 3147, has recently revealed an intrinsic $\mathrm{H} \alpha$ line with an extremely broad base (full width at zero-intensity on the order of $27000 \mathrm{~km} \mathrm{~s}^{-1}$ ), see Bianchi et al. (2019). The variation in type classification is rather straightforward to track using medium-resolution spectroscopic observations (e.g., Cohen et al. 1986; LaMassa et al. 2015). The polarization resulting from the state change, however, is more complex to predict and observe. It is rather different between the two inclinations.

In the $10^{\circ}$ case (Fig. 3a), the polarization degree is $0.3 \%$, with a parallel $\left(\Psi=90^{\circ}\right)$ polarization angle during the bright state. The low polarization is due to the almost face-on inclination of the nucleus. Starlight dilution plays a minor role here. When the flux starts to dim, the polarization degree increases to $0.4 \%$ for a very short time ( one year), then decreases sharpl to zero in a decade. The increase in polarization is due to the combined action of (1) a lower amount of direct unpolarized flux from the central engine, and (2) a constant amount of 
F. Marin and D. Hutsemékers: Polarized echoes in changing-look AGNs
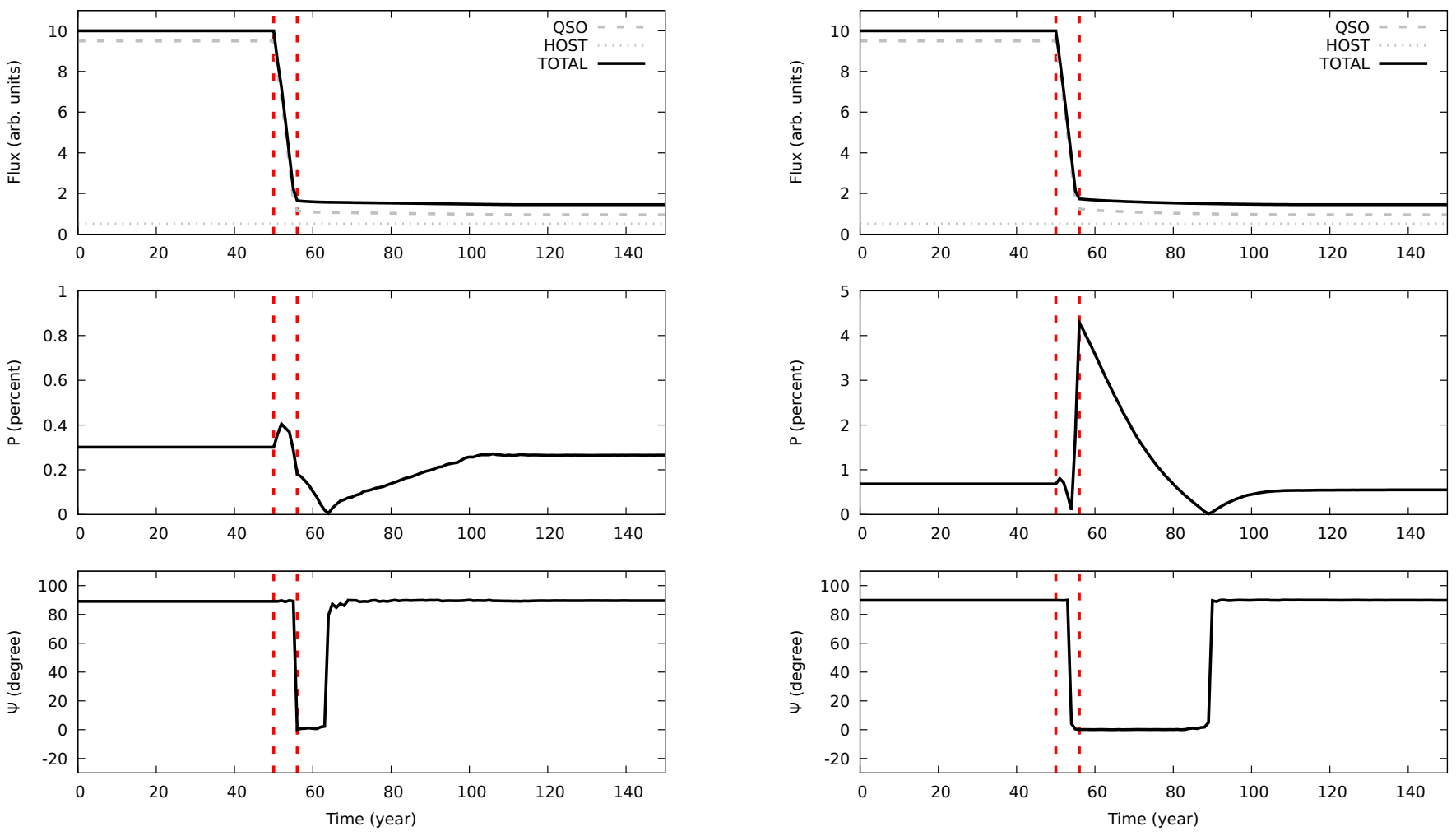

(a)

(b)
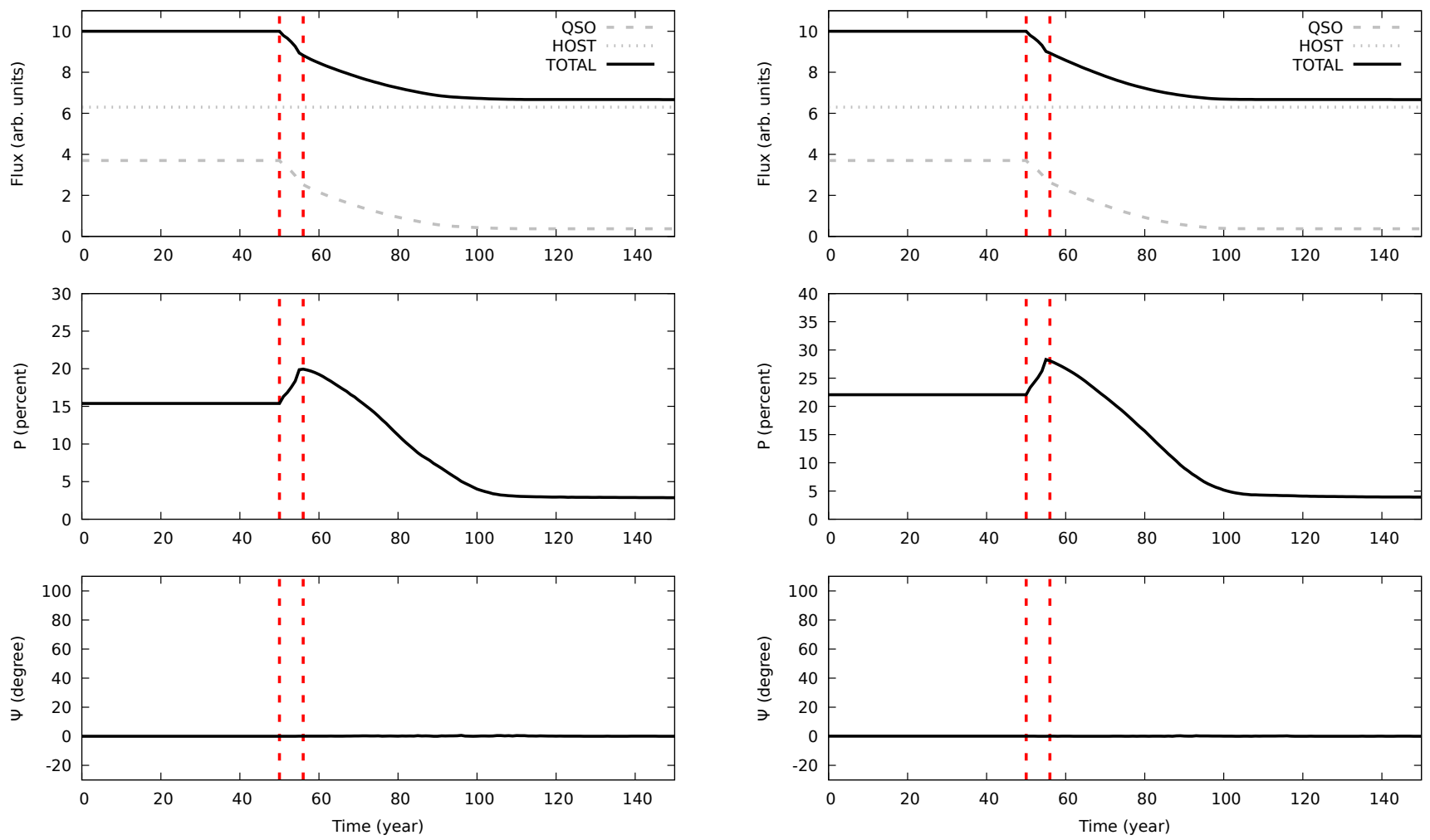

(c)

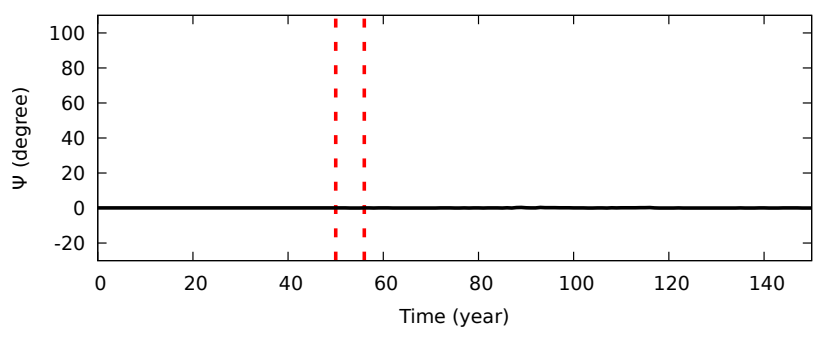

(d)

Fig. 3. Variation in normalized total flux (top panel), polarization degree (middle), and polarization position angle (bottom) from our changing-look quasar model seen along four different viewing angles: $10^{\circ}(a), 45^{\circ}(b), 60^{\circ}(\mathrm{c})$, and $80^{\circ}(\mathrm{d})$. In the total flux panels, we show the contribution of the quasar, the host galaxy, and the combination of both using gray dashed lines for the first two angles and a black solid line for the other two angles. Between the first and second long-dashed vertical red lines, the central engine dimmed by a factor of 10 in 6 years. $a$ : observer inclination $\sim 10^{\circ}$ from the polar axis of the model. $b$ : observer inclination $\sim 45^{\circ}$ from the polar axis of the model. $c$ : observer inclination $\sim 60^{\circ}$ from the polar axis of the model. $d$ : observer inclination $\sim 80^{\circ}$ from the polar axis of the model. 
reprocessed (delayed) radiation from the equatorial region. The reprocessed radiation is an echo from the past bright state. Its flux is thus higher and outshines the constant weaker unpolarized flux from the host, explaining the increase in polarization. The duration of the high-polarization peak depends on the distance of the scatterer from the source and can be used to achieve polarized reverberation mapping of the inner quasar regions, such as has been demonstrated by Gaskell et al. (2012) and Afanasiev et al. $(2014,2015)$. When the echo has faded away, the smaller number of source photons that is scattered inside the equatorial structures results in a decrease in polarization degree. At a certain point, the polarization position angle rotates and becomes perpendicu$\operatorname{lar}\left(\Psi=0^{\circ}\right)$ for less than a decade. This is due to a second polarized echo coming from the extended polar region. The ionization cones are indeed much more extended than the equatorial scattering region and have a lower electron density. The polarized echo thus takes longer to be visible. The number of photons from the past bright quasar state that have scattered inside the extended polar regions becomes larger than the ernumb of photons from the dimmed source that is seen without additional time delay. Because scattering occurs in the polar region, the polarization degree rotates and becomes perpendicular. When this secondary polarized echo has faded away, $\Psi$ rotates again as a result of the contribution of the equatorially scattered flux. The orthogonal flip of the polarization angle is responsible for the cancellation of the polarization degree, which can slowly rise again afterward. The final linear polarization degree, after the quasar has dimmed and polarized-light echoes have vanished, is about $0.27 \%$. This value is lower than during the bright state because fraction of starlight from the host is higher. It takes about 40 years for the light echoes to stop propagating inside the model, showing that polarization can track down changing-look objects for a much longer period than photometry alone. Unfortunately, the low levels of linear polarization degrees at low inclinations makes the variation in $P$ difficult to measure because observational errors on $P$ are commonly about $0.1 \%$. To facilitate polarimetric monitoring campaigns, it is therefore better to study the polarization of AGNs seen at higher inclination that represents still type $1 \mathrm{~s}$.

The variation in polarization in the case of the highest possible type 1 inclination $\left(45^{\circ}\right.$, Fig. 3a) shows a different behavior that is associated with longer timescales. The polarization degree first increases by a negligible amount as a result of the polarizedlight echo from the BEL or scattering region, similarly to the $10^{\circ}$ inclination case. Then $P$ sharply decreases as the polarization position angle rotates from parallel to perpendicular. This is due to scattering of photons from the base of the polar regions. The higher system inclination promotes higher polarization degrees ( $P$ induced by Thomson laws is cosine-squared-dependent on the scattering angle). Thus the polarization degree is dominated by a polar component and is about $4-5 \%$. Then, similarly to lower inclinations, the polarized-light echo fades away with a longer timescale because of the higher inclination (this is a geometric effect). This tells us that the transitory phase of the polarization position angle might be related to the inclination angle, allowing an independent and novel way of measuring quasar inclination. Highly inclined type 1 quasars are thus interesting targets because we can expect higher observable polarization degrees and strong time-dependent polarization signatures.

In the case of type 2 quasars (Figs. 3c and d), the situation is different but particularly interesting because we investigate a transition from a type 2 , that is, a hidden or obscured type 1 , to a true type 2 (i.e., intrinsically lacking a BEL region). Type 2 objects are often more complex to observe in polarization because starlight dilution is strong. The flux from the central quasar regions is obscured by the circumnuclear dusty component and can only be detected through reprocessed photons in the polar cones (Miller \& Antonucci 1983; Antonucci \& Miller 1985). This results in stronger host flux contributions, which commonly exceed $50 \%$ in the case of type 2 quasars (see, e.g., Zakamska et al. 2006). Because of the geometric configuration and optical depth of the various reprocessing regions in our model, only $3 \%$ of the quasar continuum flux can be detected in type 2 inclinations through polar scattering. As a consequence, Figs. $3 \mathrm{c}$ and $\mathrm{d}$ (top panels) show that during the bright state, $37 \%$ of the observed total flux is due to the AGN and $67 \%$ is due to the host. The host contribution becomes preponderant, up to $94 \%$, after the quasar has dimmed by a factor of 10 . This results in a total flux attenuation that is only a factor 1.5 lower after the change of look. The transition also takes longer, many decades, because we only see light echoes in the extended polar region. These echoes naturally carry a high polarization degree. In the case of a $60^{\circ}$ inclination, $P=15.4 \%$ in the bright state. For a $80^{\circ}$ inclination, $P=22.1 \%$. Both inclinations show a perpendicular polarization angle, the usual signature of polar scattering. When the central engine starts to dim, $P$ increases by a few percent, then slowly decreases with time, at the same pace as the total flux. The polarization position angle remains constant over time. Because the total and polarized fluxes are only due to polar scattering, these were expected behaviors. The short increase in $P$ is due to the radiative coupling of the various quasar components. Most of the polarized flux we observe is due to polar scattering in the ionization cones. However, a smaller fraction is also due to scattering of photons inside the equatorial dust funnel. The inclination of the quasar means that a small fraction of radiation can escape by (back)scattering from the dust wall opposite to the observer's side (Marin et al. 2012). This low photon flux carries a parallel polarization that weakly dilutes the final perpendicular polarization we observe. When the central engine dims, this equatorial contribution decreases more rapidly than the polar echo because of the different spatial extensions of the components. This results in less dilution, hence a marginally higher polarization degree. When the quasar has dimmed by a factor 10 , the system stabilizes and the polarization degree decreases with time. The final polarization we observe is much lower than in the bright phase because the contribution from host starlight is higher. In the $80^{\circ}$ inclination case, $P$ ends at $3.9 \%$ (Fig. $3 \mathrm{~d}$, middle panel). Our study of changing-look quasars with inclinations higher than $45^{\circ}$ demonstrates that the transition of a type 2 , that is, a hidden or obscured type 1 to a true type 2 can be detected with polarimetry.

\subsubsection{Brightening quasars}

We now investigate the opposite trend in changing-look quasars: a brightening nucleus. We present our results in Figs. $4 a$ and $b$ for type 1 objects and in Figs. $4 \mathrm{c}$ and d for type 2 objects. The flux also varies by a factor 10 and the models are exactly the same. In the following section we do not describe the total flux plots because they are the exact opposite to the dimming cases. The polarization plots, however, show several different features.

In the case of type 1 objects (Figs. $4 \mathrm{a}$ and b), the polarization degree shows a rapid but small variation in $P$, while $\Psi$ remains constant. Most of the changes in $P$ occur during the brightening phase of the quasar and result in a partial decrease in polarization followed by a longer increase (a few years) and a smooth decrease with time. The sharp decrease in $P$ immediately follows the brightening of the central engine and is easily 
explained by the large amount of unpolarized radiation that is created by the source. It takes less than a year for the brighter continuum flux to reach the equatorial scattering region, resulting in an enhanced production of polarized photons from that region. This is the reason for the subsequent increase in $P$ during the brightening phase. When the nucleus reaches a stable luminosity, the polarization stabilizes with a short delay due to the distance between the source and the reprocessing regions inside the nucleus. Because the brightening of the quasar enhances the reprocessed polarized flux from the equatorial regions before the reprocessed polarized flux from the polar winds, no variations in $\Psi$ are detected or expected. The main difference between the $10^{\circ}$ and $45^{\circ}$ inclinations is the value of $P$ (which remains low, below $1.5 \%$ at best) and the time it takes for the polarization degree to stabilize. Similarly to the dimming cases, polarimetry can probe brightening phases, but the signatures are less prominent.

In the time-dependent polarization of type 2 changing-look quasars (Figs. 4c and d), we observe a polarization degree that slightly decreases when the brightening of the central engine sets on, then a smooth and slow increase in $P$ for several decades, following the total flux. The polarization position angle remains constant and perpendicular to the projected symmetry axis of the system, as expected from type 2 AGNs. The time-dependent behavior of $P$ shows in more detail that $P$ decreses during the first years to the enhanced production of equatorial, backscattered photons from the dust funnel opposite to the observer, as we discussed in the previous section. This stronger polarized flux, carrying a parallel polarization angle, dilutes the observed polarization during the first years. The contribution of equatorial photons then diminishes as the stronger polarized flux from the polar winds (situated at a larger radial distance from the source than the inner dust funnel) is visible above the circumnuclear dust horizon. The polarization then steadily increases together with the total flux up to a constant and high $(>10 \%)$ value. The two cases $\left(60^{\circ}\right.$ and $\left.80^{\circ}\right)$ are only discernible by their final polarization degree.

\subsubsection{Comparison with changing-look Seyferts}

We finally investigate the case of Seyfert galaxies. We fixed the host contribution to $50 \%$ of the total flux in type 1 views according to the average host contribution observed in nearby lowluminosity AGNs (Kauffmann et al. 2003). When the nucleus is obscured at high inclinations (type 2 view), up to $97 \%$ of the AGN radiation comes from the host galaxy. The main result we obtained (see Fig. 5) is that the polarization time series of changing-look Seyfert galaxies are very similar to those of quasar, but are far more strongly diluted because starlight from the host galaxy is stronger. This is true for both dimming and brightening phase, therefore we only show the dimming case. Figure 5 shows that the main difference in terms of polarization occurs at very low inclinations $\left(i \sim 10^{\circ}\right)$. At the onset of the dimming phase, the polarization degree experiences a sharp decrease that is associated with an orthogonal flip of the polarization angle. $P$ then rapidly increases before it returns to a stability period after a second flip of $\Psi$. This behavior has been observed for higher inclinations (see, e.g., the $45^{\circ}$ case). We observe this in low-inclined changing-look Seyferts but not in low-inclined changing-look quasars because the host contribution is stronger. In both cases the polarization degree emerging from equatorial or polar scattering is low at $i \sim 10^{\circ}$, but in quasars the central engine largely dominates the unpolarized flux from the host so that the polarization degree is less diluted. We also observe longer orthogonal flip periods of the polarization position angle for Seyfert 1 inclinations. The previous simulations showed that the reversal in polarization angle takes place when the polarization of the polar echo becomes equal to the equatorial polarization. These two polarizations are fixed by the brightness of the central nucleus, which in the case of Seyfert galaxies is much lower than for quasars. We observe the polar echo for a longer period because its polarized flux overwhelms the unpolarized flux from the host and the weaker polarized flux from the equatorial scattering region for a longer time. This explains the about 15 years longer orthogonal flip of the polarization position angle in Fig. 5b. For type 2 viewing angles, there is no difference other than a stronger dilution of $P$ for lower luminosity AGNs. Our modeling shows that quasars are the best observational targets for monitoring fast and strong variations in polarization after a change of look.

\section{Application to Mrk 1018}

We showed that polarimetry is a powerful method for following the evolution of CLAGNs, in particular when the state transition is due to variations in mass accretion rate. Unfortunately, no multi-epoch polarimetric data are available so far for a quasar or a Seyfert galaxy that has shown a state transition that might be associated to intrinsic variation and is therefore likely to generate echoes. To support such eagerly awaited observations, we selected the best-documented case, the changing-look Seyfert galaxy Mrk 1018, and applied our numerical tool to make quantitative predictions for a future observing campaign.

Mrk 1018 is a type $1 \mathrm{AGN}$ situated at a Hubble distance of 183.7 Mpc (heliocentric redshift $=0.04244$ ). It is a peculiar target because it is one of the few low-luminosity AGNs (Seyfert galaxy) known to have transitioned many times across the spectral sequence. Mrk 1018 varied from type 1.9 to 1 between 1979 September and 1984 January (Cohen et al. 1986), then returned again to type 1.9 in 2015 (McElroy et al. 2016). The AGN is currently stabilizing at low nuclear optical fluxes since 2017 (Krumpe et al. 2017), as seen in Fig. 6 (top panel). These changes of look are explained by a variation in the accretion rate onto the SMBH (Husemann et al. 2016).

The very recent state transition of Mrk 1018 makes it an excellent and priority target for polarimetric observations in order to assess the origin of the change of look. Unfortunately, only one polarimetric observation of Mrk 1018 has been made so far. It was made on 9 February 1986. At that time, Goodrich (1989) reported a polarization degree of $0.28 \pm 0.05 \%$ in the 4180-6903 $\AA$ band. It is difficult to use this measurement because the reported polarization degree is very low; this might be due to interstellar polarization from the host galaxy or to foreground dust in our Galaxy. The associated polarization position angle $\left(\theta=165.1 \pm 5.2^{\circ}\right)$ is unlikely to be meaningful either becaue we do not know the origin of the polarization. In addition, we do not know the position angle of the (sub)parsec scale radio structure in Mrk 1018 either. Milliarcsecond angular resolution radio imaging of Mrk 1018 with the Very Low Baseline Array (VLBA) does not resolve the core-jet structure of the AGN (Pérez-Torres, priv. comm.). We therefore do not know if Mrk 1018 had a parallel or perpendicular polarization angle in 1986. New polarimetric measurements of $\theta$ might indicate whether the polarization angle has rotated since then.

We modeled Mrk 1018 using the same general AGN geometry as in Sect. 2. We fixed the host galaxy contribution during the bright (pre-2011) and dim (post-2016) states of Mrk 1018 using the luminosity ratio of the nucleus to the host galaxy as 

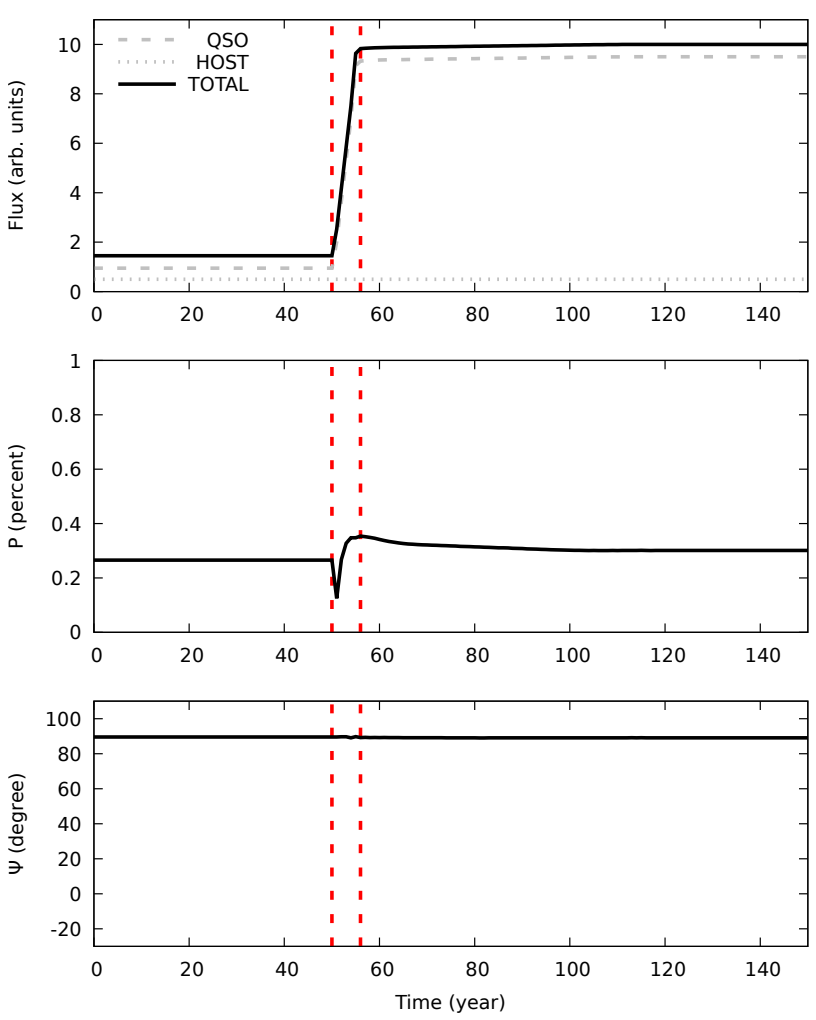

(a)
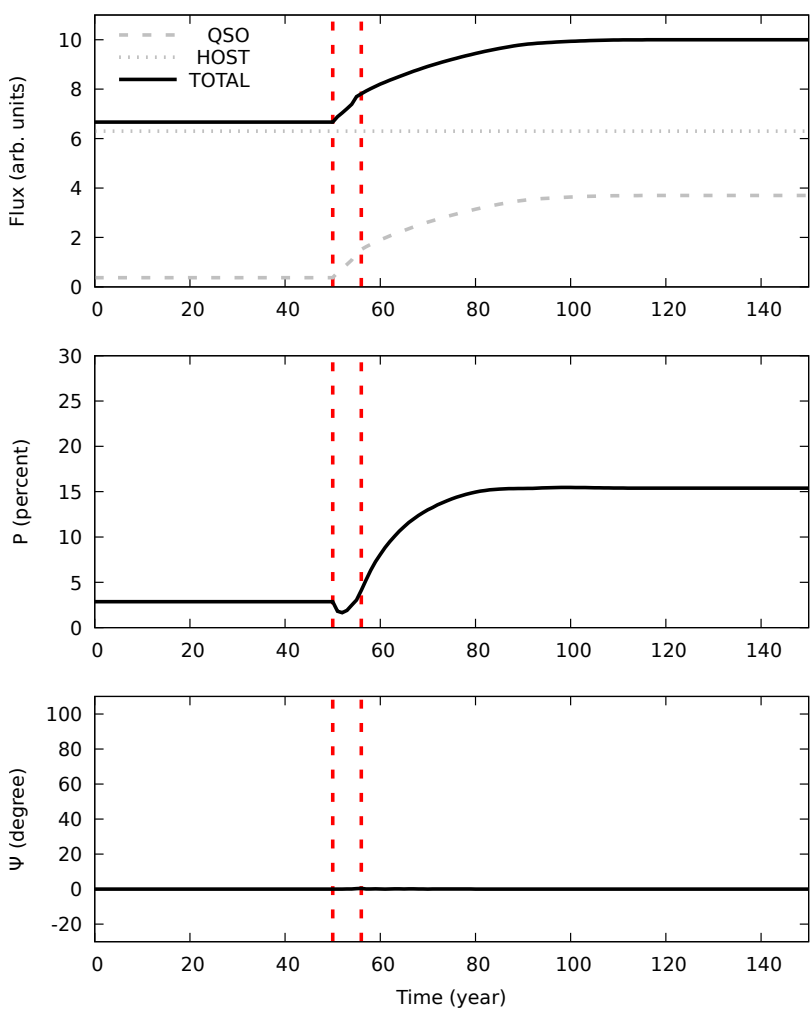

(c)
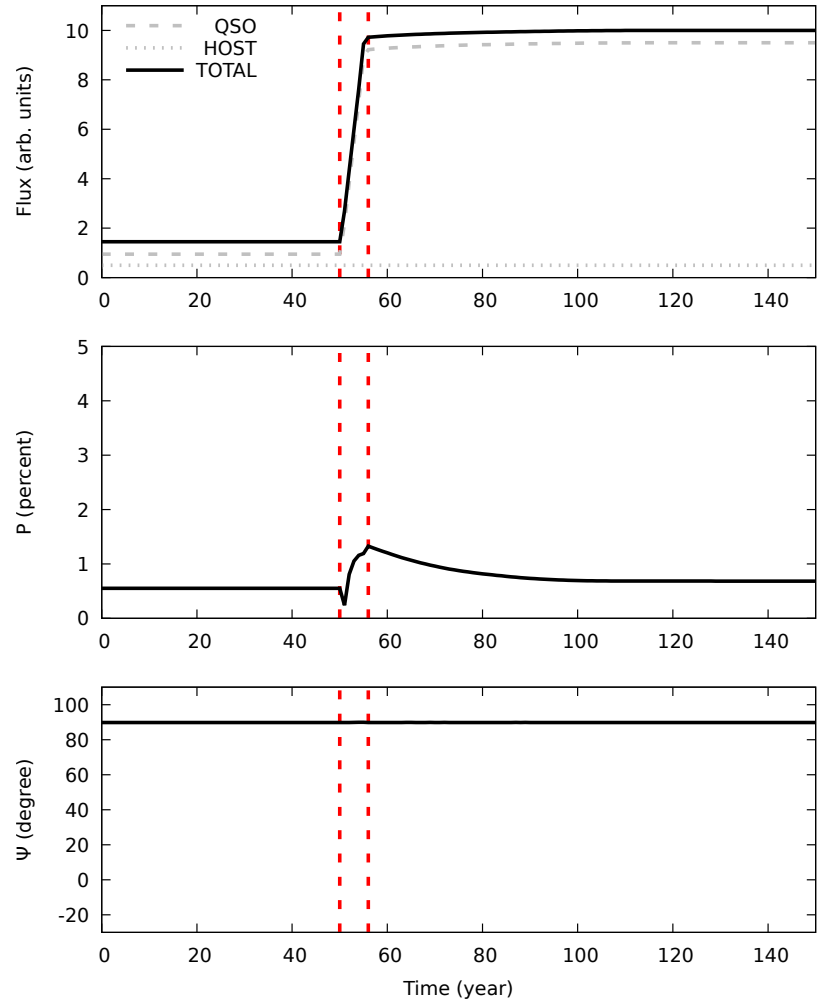

(b)
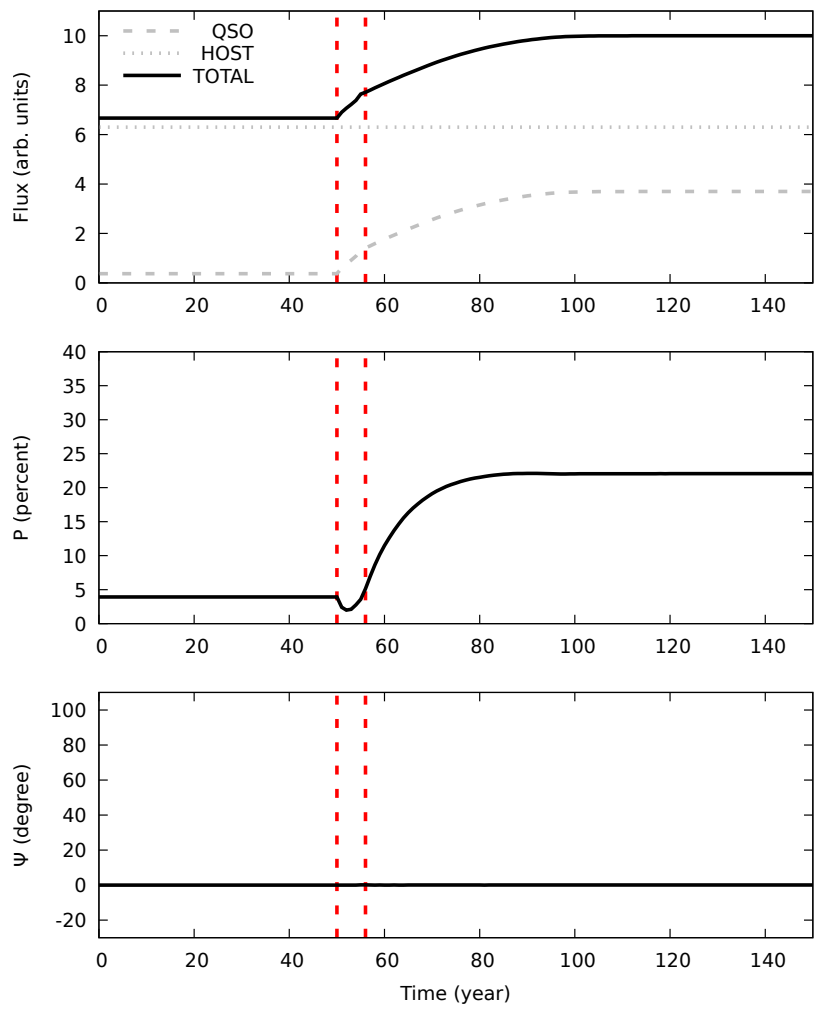

(d)

Fig. 4. Variation in normalized total flux (top panel), polarization degree (middle), and polarization position angle (bottom) from our changing-look quasar model seen along four different viewing angles: $10^{\circ}(a), 45^{\circ}(b), 60^{\circ}(c)$, and $80^{\circ}(d)$. In the total flux panels, we show the contribution of the quasar, the host galaxy, and the combination of both using gray dashed lines for the first two angles and a black solid line for the other two angles. Between the first and second long-dashed vertical red lines, the central engine brightened by a factor of 10 in 6 years. $a$ : observer inclination $\sim 10^{\circ}$ from the polar axis of the model. $b$ : observer inclination $\sim 45^{\circ}$ from the polar axis of the model. $c$ : observer inclination $\sim 60^{\circ}$ from the polar axis of the model. $d$ : observer inclination $\sim 80^{\circ}$ from the polar axis of the model. 
F. Marin and D. Hutsemékers: Polarized echoes in changing-look AGNs
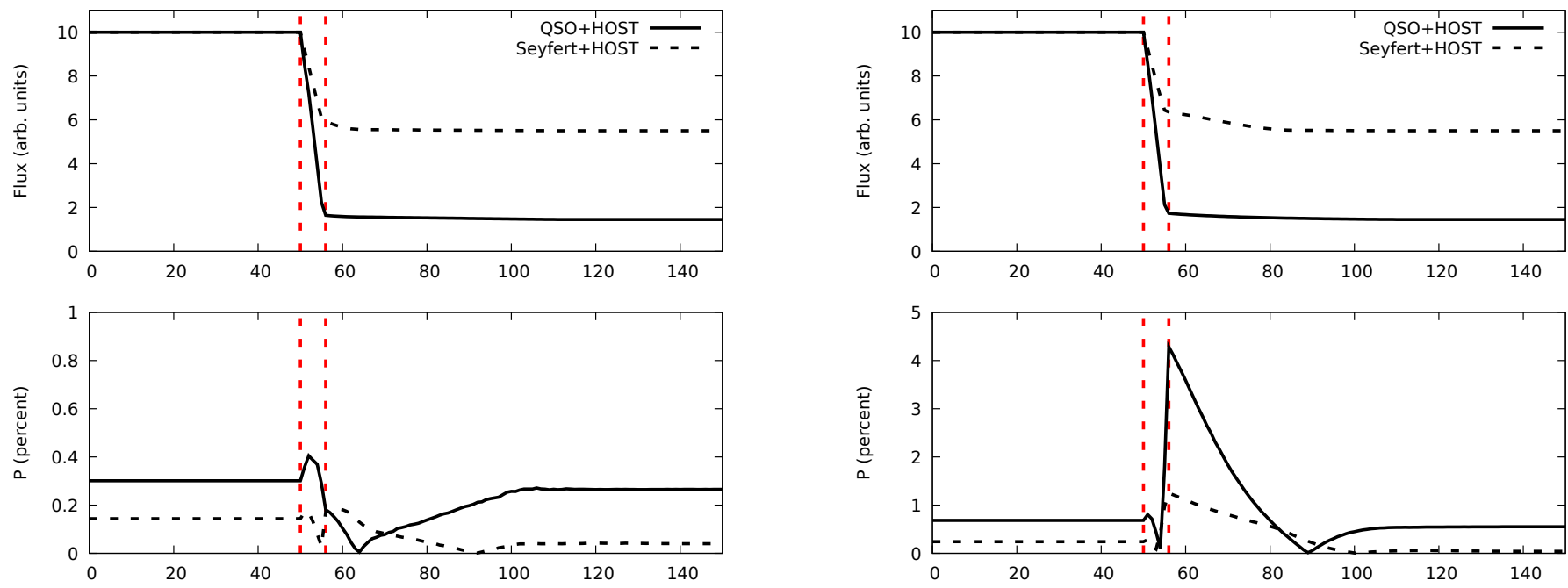

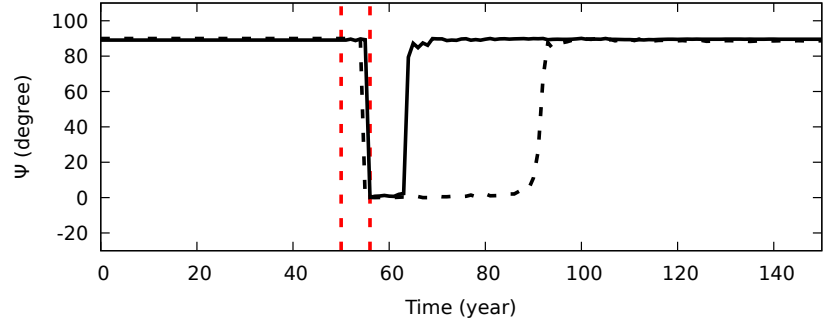

(a)
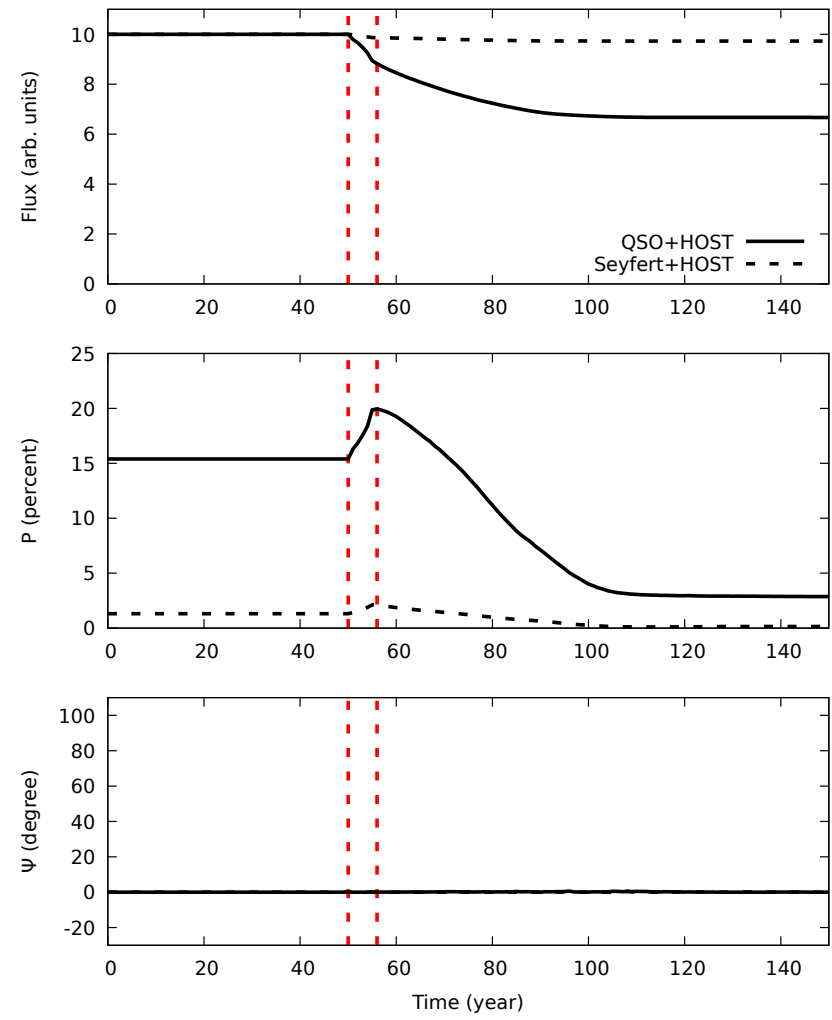

(c)

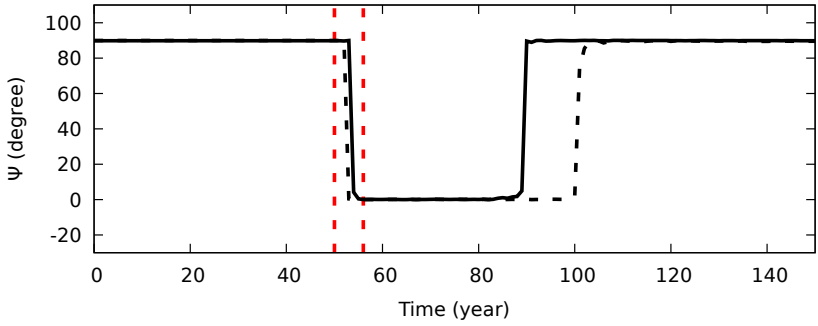

(b)
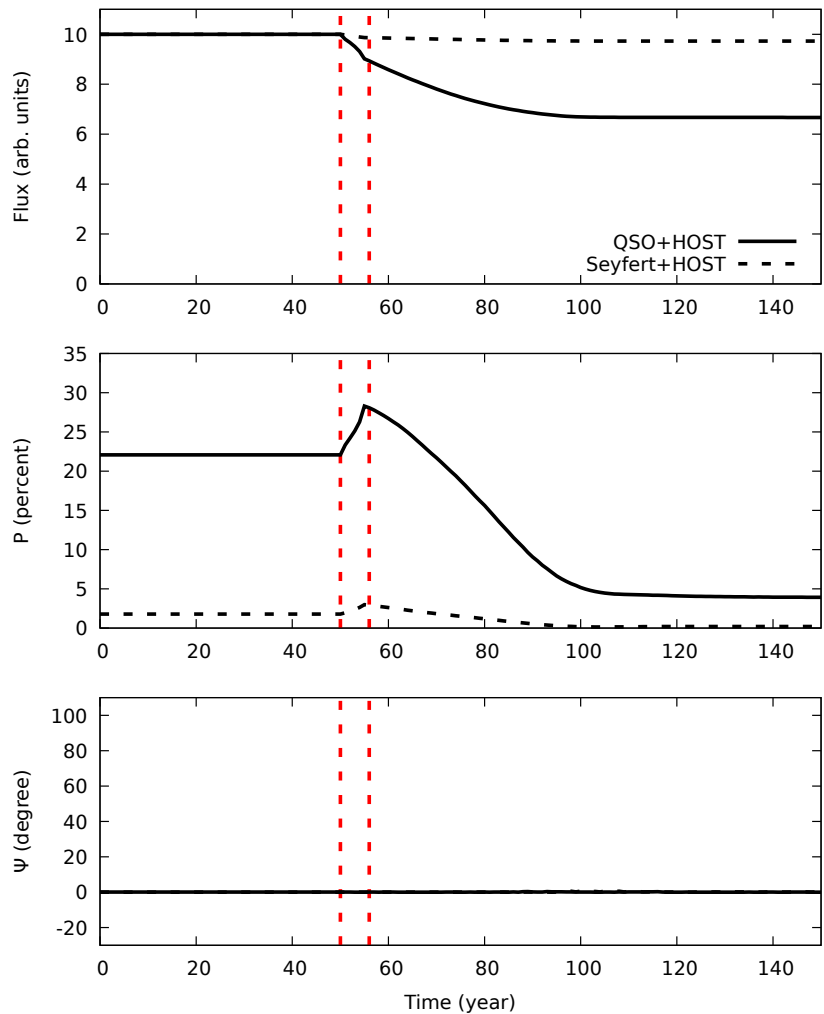

(d)

Fig. 5. Comparison between the polarization time series of changing-look quasars (solid line) and Seyfert galaxies (dashed lines). Only the relative contribution of the host galaxy to the total and polarized type 1 fluxes is different: 5\% in the quasar case, and 50\% for Seyfert galaxies. The rest of the panel description is similar to the caption of Fig. 3. $a$ : observer inclination $\sim 10^{\circ}$ from the polar axis of the model. $b$ : observer inclination $\sim 45^{\circ}$ from the polar axis of the model. $c$ : observer inclination $\sim 60^{\circ}$ from the polar axis of the model. $d$ : observer inclination $\sim 80^{\circ}$ from the polar axis of the model. 

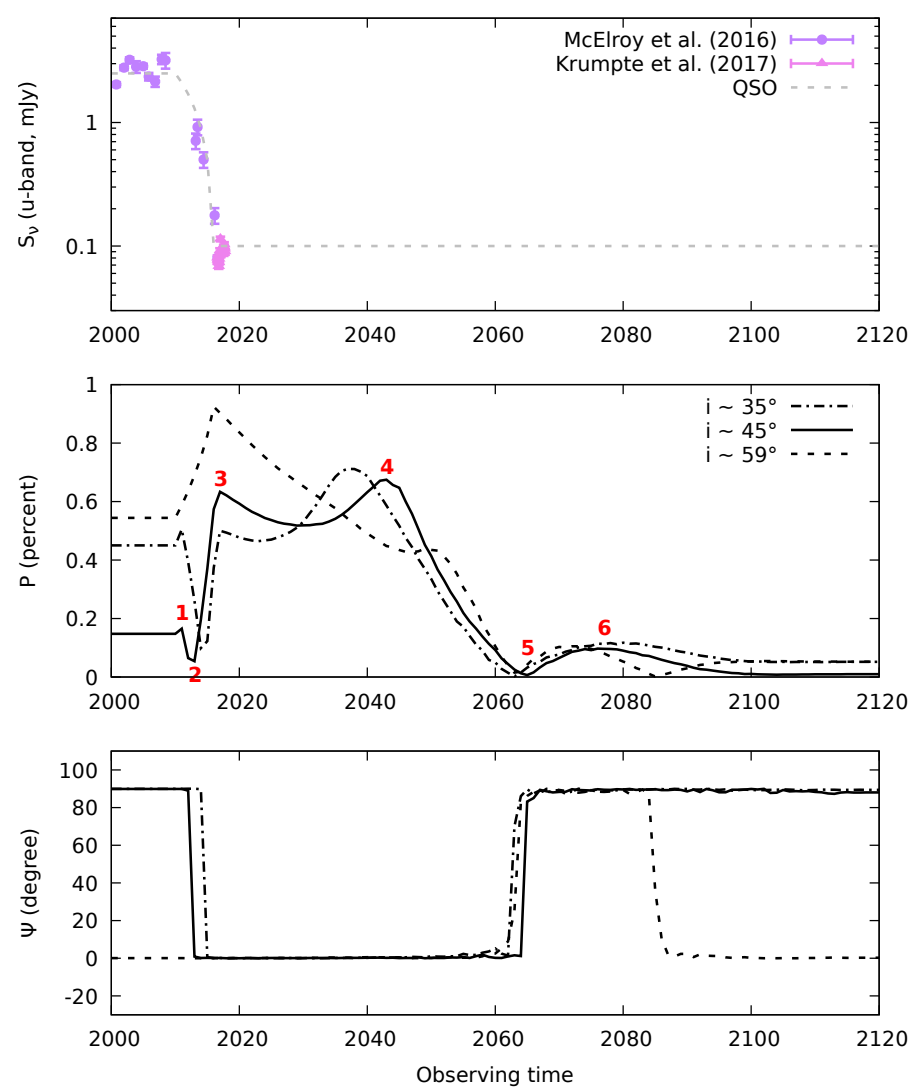

Fig. 6. Top panel: time series of archival $u$-band flux density $S_{v}$ for the AGN in Mrk 1018 (McElroy et al. 2016; Krumpe et al. 2017). The host galaxy contribution has been carefully removed by the authors. We plot in gray the flux function we used in our simulation to mimic the dimming of the AGN (host contribution removed). Middle and bottom panels: model predictions for the polarization degree and polarization angle for different nuclear inclinations. See text for an explanation of the red numbers.

a function of epoch presented in Kim et al. (2018). The authors analyzed multi-epoch Sloan Digital Sky Survey (SDSS) $g$-band and Swift $B$-band imaging data and derived a luminosity fraction $L_{\mathrm{AGN}} / L_{\text {host }} \sim 0.4$ in the bright state and $\sim 0.03$ in the $\operatorname{dim}$ state. We allowed the nucleus to decreases its flux by a factor 25 , such as observed by McElroy et al. (2016). Finally, we observed the system along a specific range of viewing angles. According to Walton et al. (2013), the AGN inclination is $45_{-10}^{+14} \mathrm{deg}$. This value was found using reflection-based modeling of the broadband X-ray spectrum of Mrk 1018 and remains the only estimate for Mrk 1018 so far (Marin 2016). We therefore investigate in Fig. 6 three inclinations: $35^{\circ}, 45^{\circ}$, and $59^{\circ}$, which covers the entire inclination range derived by Walton et al. (2013). We note that at $i=59^{\circ}$, the line of sight crosses the equatorial dust. This would make Mrk 1018 a type-2 Seyfert galaxy at all epochs but, for the academic interest of the study, we also consider this inclination.

We show in the top panel of Fig. 6 the observed timedependent $u$-band flux density $S_{v}$ of Mrk 1018 (in mJy). The source flux function we used was set to become fainter by a factor of 25 between 2011 and 2017 and is shown in gray. We allowed the system to reach a stable configuration following 2017 for the next century but we note that a new state transition might very well occur in the future. For simplicity, we only show one transition here. In the middle and bottom panels of Fig. 6 we show the polarization degree and polarization

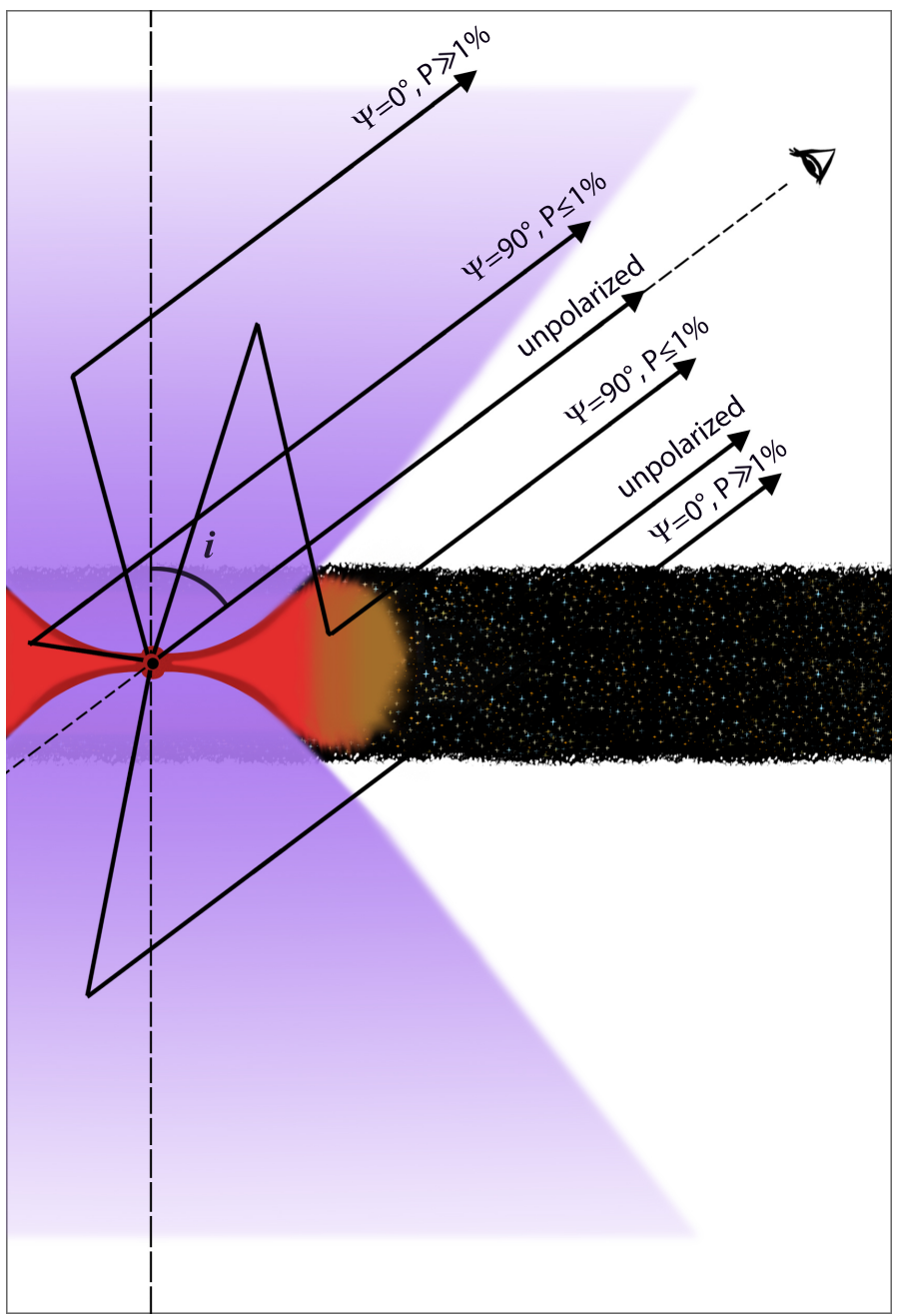

Fig. 7. Illustration of the various paths taken by the photons for a typical type 1 viewing angle $\left(i \sim 45^{\circ}\right)$. The expected polarization signature of emission or reprocessing regions is indicated. The breaks in the light rays are due to scattering onto electrons or dust grains. See text for details.

position angle, respectively. For all inclinations, the polarization degree appears low, below $1 \%$ because of the strong dilution by the host galaxy. These polarization levels are in good agreement with the unique measurement made by Goodrich (1989). The three different inclinations are displayed using different line types. The polarization behavior is more complex than what was shown in the previous sections. The main reason for this increasing complexity is the stronger variation in the core flux (a factor 25 instead of the factor 10 explored in the previous sections) that will generate secondary echoes. To better understand the various polarization signatures we expect to observe, we report the various possible trajectories of photons in Fig. 7 and number the principal features in red in Fig. 6 (middle panel) for the $\sim 45^{\circ}$ inclination. We give an explanation of each feature below.

1. When the dimming sets on, we observe an almost negligible increase in $P$. This feature has been explained for Figs. 3a and $b$ and corresponds to the delayed response of the equatorial scattering region to the nuclear dimming.

2. The local dip in $P$ is due to the orthogonal flip of $\Psi$. The superposition of parallel polarization from the equatorial regions and perpendicular polarization from light echoes in the polar winds cancel each other out. 
3. The maximum of perpendicular polarization is due to the propagation of the bright-light echo inside the upper part of the biconical outflow. At this point, the polarized echo starts to fade away.

4. The second maximum of the perpendicular polarization is due to the propagation of the bright-light echo inside the lower part of the biconical outflow. The delay between the two polar echoes is a geometric effect that is due to the inclination of the AGN. The second light echo then starts to fade away as well.

5. The second dip in $P$ is due to the second orthogonal flip of $\Psi$. The dominance of polar-light echoes ends and the observed polarization again becomes parallel.

6. A final bump in polarization is detected before $P$ resumes a stable level (which is highly diluted by the strong starlight from the host). This bump is due to multiple scattering of photons from the light echo in the polar regions that traveled back to the AGN core and scattered onto the external surfaces of the circumnuclear dust (Marin et al. 2012; Grosset et al. 2018).

The same reasoning holds for the $35^{\circ}$ inclination. In the case of $i=59^{\circ}$, the change in polarization is similar to what was described in Figs. 3c and d. In addition, a secondary polarized echo from the lower part of the biconical structure is visible in about 2050. The scattering of perpendicularly polarized photons from the polar winds on the external surfaces of the circumnuclear dust is highlighted by a new flip of the polarization angle in about 2085. Interestingly, Figs. 3 and 4 the polarization features associated with the propagation of the light echo inside the lower part of the biconical outflows are absent. Using STOKEScomputed polarization maps, Marin et al. (2012) showed that most of the observed polar flux is due to scattering within the first parsecs of the biconical outflow. At type 1 inclinations, the base of the lower part of the biconical outflow is hidden by the distribution of equatorial dust. The photon flux from the southern wind base is therefore mainly absorbed by the optically thick dusty torus, and radiation scattered at larger distances is relatively weak. However, if the flux variation is extreme, the light echoes are stronger and more photons can reach more distant parts of the lower biconical outflow that are not hidden by the thick dusty torus. This stronger and delayed echo of polarized flux explains why we detect an additional polarization signature when the continuum source has dimmed by a very large factor.

Ultimately, we demonstrated that a polarimetric campaign targeting Mrk 1018 over the next decade could tell us much about the physics and morphology of the innermost components of nearby AGNs. Even if the polarization is strongly diluted by the host starlight, polarization variability can be measured independently of the contamination, provided that the instrumentation is accurate enough. We discuss this point in more detail in Sect. 4.1.

\section{Discussion}

Our modeling has demonstrated that changing-look AGNs are expected to present strong and long polarized echoes that are associated with both a dimming or brightening phase. While the total flux variation lasts for only a few years, the polarized echo lasts for decades. This increases our chances of detecting and characterizing the accretion processes and the geometry of the various reprocessing regions in quasars and Seyfert galaxies. In Sect. 4.1 we discuss the observational constraints we can place on nearby and distant AGNs while estimating the relative importance of model degeneracies. We also report in Sect. 4.2 the most interesting cases for future polarimetric observations in order to better understand the underlying causes of the accretion rate variability that is responsible for CLAGNs.

\subsection{Observational constraints and model degeneracies}

After the onset of the dimming phase in type 1 CLAGNs, a slight increase in $P$ is followed by a strong decrease that is associated with an orthogonal flip of the polarization position angle (see Figs. 3a and b). As we described, the increase in $P$ is due to the continuum echoing onto the equatorial scattering region (parallel $\Psi$ ), which is soon substituted by the polarized echo on the polar winds (perpendicular $\Psi$ ). A triggered polarization monitoring of a CLAGN currently undergoing a state transition from type 1 to type 2 may very well measure the delay between the dimming and the light echo from the equatorial scattering region and/or the polar structure. This time information could then be translated directly into geometric constraints in the form of upper limits on the inner radius of the two reprocessing regions. The inferred inner radii would be dependent on the optical depth of the specific scattering region, but we already know that the polar winds and the equatorial scattering region are not highly opaque (Osterbrock 1991). In addition, the orthogonal flip of $\Psi$ would clearly indicate which scattering region dominates in terms of flux. Dilution by the host does not interfere with these observational constraints (see, Fig. 5b), it can only make the observation more time-consuming because of the lower expected polarization degree in Seyfert galaxies. The duration of the orthogonal flip in polarization angle is directly related to the inclination of the nuclear system. The longer the flip, the more inclined the object. However, this needs to be carefully modeled. Figure 5a clearly demonstrates that a high host or AGN flux ratio may also increase the duration of the $\Psi$ reversal. In such cases, it will be mandatory to determine the host contribution before the true nuclear inclination of the AGN is determined. This means that a new and spectroscopically independent measurement of the global AGN inclination can be achieved in CLAGNs. If no orthogonal flip of $\Psi$ is observed, it means that the system is seen along an equatorial viewing angle, that is, the observer's line of sight crosses the circumnuclear dust region. The statistical counting of CLAGNs with and without polarization angle reversals would bring crucial information on the average half-opening angle of the dusty torus and help determine its formation process. If the torus results from mass transfer from the host to the AGN, its geometry is expected to be flatter than when the torus is in fact a wind that flows out at low velocities from the AGN to the host (see, e.g., Krolik \& Begelman 1988; Konigl \& Kartje 1994; Lovelace et al. 1998; Czerny \& Hryniewicz 2011; Dorodnitsyn \& Kallman 2012). Moreover, because CLAGNs are detected up to redshifts higher than 0.8 (Graham et al. 2020), it will be possible to determine whether the geometric configuration of the torus evolves with redshift and luminosity.

A final remark must be added concerning model degeneracies in our analysis. The features detected in the polarization time series are model dependent. As we mentioned, the AGN or host flux ratio plays a role, but this may be counterbalanced by dedicated measurements. The geometry and optical depth of the AGN reprocessing components will mainly affect the delays seen in polarized echoes. They do not play a major role in the observed degrees of polarization, at least for type 1 objects (Marin et al. 2012). The difference is more noticeable for equatorial lines of sight, where the optical depth and half-opening angle of the polar winds will provide most of the polarized flux. Fortunately, the extended polar winds are not too difficult 
to resolve spatially, at least in nearby AGNs. From narrow-line emission intensity and widths, constraints on the electron optical depth can be derived while medium-resolution intensity [O III] maps and long-slit spectra can provide an insight into the wind half-opening angle. Fischer et al. $(2013,2014)$ used this technique to determine the inclination of the extended biconical outflows with respect to the plane of the sky with a $5^{\circ}$ precision. The main source of degeneracies remains the spatially unresolved innermost AGN components. Although interferometry is now able to probe the subparsec-scale regions in the most luminous and closest AGNs (Gravity Collaboration 2018), such observations will remain impossible for the vast majority of AGNs for the foreseeable future. Careful modeling of observational (spectro)polarimetric and photometric data will help to reduce the number of free parameters, but the uncertainties associated with the method remain to be tested.

\subsection{Past polarimetric observations of CLAGNs}

In the case of changing-look quasars, two publications currently report the measurement of associated optical polarization: Hutsemékers et al. (2017, 2019). The first paper only considered one CLAGN, J101152.98+544206.4, a changing-look quasar $(z=0.246)$ discovered by Runnoe et al. (2016), which transitioned from a bright state in 2002 to a faint state in 2017. The 2017 optical linear continuum polarization degree of this object is $0.15 \pm 0.22 \%$, consistent with null polarization, therefore it was not possible to estimate its polarization angle. The very weak (most probably null) polarization of J101152.98+544206.4 tells us about the probable inclination of this quasar (close to pole-on, Marin 2017). According to our simulations, the polarized-light echo of the state transition is currently propagating, but because of the very low expected polarization degree (see Fig. 3a), detecting polarization variations is difficult. The second paper presents a more promising source. As shown in Hutsemékers et al. (2019), polarization echoes can explain the high polarization $\left(P=6.8 \%, \theta=71 \pm 3^{\circ}\right)$ observed in the changing-look quasar J022652.24-003916.5 $(z=0.625)$ that is presently in a faint state, assuming that this quasar is seen at intermediate inclination. Our simulations (see Fig. 3b) show that a regular decrease in polarization degree is expected during the next decades, ultimately with a reversal of the polarization angle. Because of the high polarization degree of the object, a monitoring campaign could easily prove or disprove the accretion rate variation scenario and narrow down the real inclination of the quasar, depending on the exact date of the expected orhtogoanl flip of the polarization position angle. In the same publication, the low polarization degrees $(\leq 1.10 \%)$ measured for the other changing-look quasars in a type 2 state are also compatible with the existence of polarization echoes if these objects are seen at low inclinations. Variations are expected, but lie below the accuracy of available measurements. In these objects, a reversal of the polarization angle is probably the most sensitive way to unveil to presence of polarization echoes.

In Seyfert galaxies, changes of look are explained by variation in accretion rate in at least three of them: Mrk 1018 (Husemann et al. 2016, Mrk 590 Denney et al. 2014, and NGC 2617 Shappee et al. 2014). Polarization echoes can thus be reasonably expected. Unfortunately, almost no archival polarization data are available for these objects. As we mentioned, the polarization of Mrk 1018 was measured in 1986 by Goodrich (1989) when the AGN was in a type 1 state: $P=0.28 \pm 0.05 \%$, and $\theta=165.1 \pm 5.2^{\circ}$. The polarization of Mrk 590 was measured in 1976 by Martin et al. (1983) when the AGN was in a type
1 state: $P=0.32 \pm 0.30 \%$, and $\theta=105.9 \pm 26.6^{\circ}$. However, this measurement is unlikely to be useful because the reported polarization degree $P$ is on the same order as $\sigma_{\mathrm{P}}$ so that the polarization angle is undefined ( $P$ is a positive quantity with nonGaussian errors; Simmons \& Stewart 1985). Finally, the polarization of NGC 2617 was measured in 1998 by Wills et al. (2011) when the AGN was in a type 2 state: $P=0.43 \pm 0.15 \%$, and $\theta=16 \pm 10^{\circ}$. In the two later cases, if the observed polarization is really due to scattering of nuclear light, the low polarization degrees might indicate that the AGNs are seen at low inclinations, but strong dilution by the host can also be expected. Estimating the inclination of AGNs is complex (Marin 2016), and the values we have for Mrk 1018 (45 $5_{-10}^{+14}$, Walton et al. 2013) and Mrk $590\left(17.8_{-5.9}^{+6.1}\right.$, Wu \& Han 2001) might very well be revised based on a polarization monitoring of those objects. Overall, there has been no new polarization measurement of any changing-look Seyfert galaxies since the end of the 1990s, while the phenomenon of type transition has become commonly known. This fact demands new polarization measurements of all CLAGNs in order to create a database for future analyses.

\section{Conclusions}

We have undertaken a series of single-wavelength simulations to predict the polarization time variations of CLAGNs. We investigated both the low-luminosity nearby cases (Seyferts) and the high-luminosity distant ones (quasars). We simulated a dimming or a brightening scenario of the central engine by several times in few years and observed the propagation of polarized-light echoes on timescales that are much longer than what photometry alone can reveal. The time-dependent polarization signatures, both in terms of polarization degree and angle, highlight the different AGN components at various scales (from subparsec to kiloparsec regions). They allow us to place constraints on the inner radii and optical depth of the various reprocessing components. An orthogonal flip of the polarization position angle in type 1 CLAGNs provides a new method for estimating the inclination of the nucleus, independently of the associated spectroscopic signatures. We applied our simulations to a specific case, Mrk 1018, which is one of the best-documented CLAGN cases. We predict its time-dependent polarization signatures after the recent dimming by a factor 25 of the AGN flux. Our numerical analysis demonstrates that crucial information on the innermost structure and on the inclination angle of the entire system may be gained by implementing a new campaign of polarization monitoring of CLAGNs. In particular, the spectropolarimetric mode of large-class telescopes would greatly improve our understanding of the physical processes that are responsible for the dimming or brightening of CLAGNs. A direct comparison between the polarization spectra of the nucleus and of the large-scale polar region would help us to follow the evolution of the light echo. Such a program would help constrain the scenario of the mass accretion rate variation and place strong upper limits on the inner radii of the equatorial and polar scattering regions even in spatially unresolved objects. In addition, new polarization measurements of CLAGNs are crucial for determining whether unification by orientation is still dominant in AGN models. We therefore strongly advocate a renewed observational effort to fully characterize the spectroscopic and imaging polarization properties of CLAGNs.

Acknowledgements. The authors would like to thank Robert "Ski" Antonucci for refereeing this paper and bringing many insightful comments. The authors are also grateful to Miguel Á. Pérez-Torres for discussing his private VLBA and 
EVN observations of Mrk 1018. FM would like to thank the Centre national d'études spatiales (CNES) who funded his post-doctoral grant "Probing the geometry and physics of active galactic nuclei with ultraviolet and X-ray polarized radiative transfer". DH is senior research associate F.R.S.-FNRS. Finally, the authors are grateful to Jules Garreau for his artworks of the AGN model.

\section{References}

Afanasiev, V. L., Popović, L. Č., Shapovalova, A. I., Borisov, N. V., \& Ilić, D. 2014, MNRAS, 440, 519

Afanasiev, V. L., Shapovalova, A. I., Popović, L. Č., \& Borisov, N. V. 2015, MNRAS, 448, 2879

Antonucci, R. R. J. 1984, ApJ, 278, 499

Antonucci, R. 1993, ARA\&A, 31, 473

Antonucci, R. 2002, in Astrophysical Spectropolarimetry, eds. J. Trujillo-Bueno, F. Moreno-Insertis, \& F. Sánchez, 151

Antonucci, R. 2012, Astron. Astrophys. Trans., 27, 557

Antonucci, R. R. J., \& Miller, J. S. 1985, ApJ, 297, 621

Antonucci, R., Hurt, T., \& Miller, J. 1994, ApJ, 430, 210

Assef, R. J., Stern, D., Noirot, G., et al. 2018, ApJS, 234, 23

Bianchi, S., Antonucci, R., Capetti, A., et al. 2019, MNRAS, 488, L1

Capetti, A., Macchetto, F., Axon, D. J., Sparks, W. B., \& Boksenberg, A. 1995, ApJ, 452, L87

Cohen, R. D., Rudy, R. J., Puetter, R. C., Ake, T. B., \& Foltz, C. B. 1986, ApJ, 311,135

Czerny, B., \& Hryniewicz, K. 2011, A\&A, 525, L8

Czerny, B., Li, Y.-R., Sredzinska, J., et al. 2017, Front. Astron. Space Sci., 4, 5

Denney, K. D., De Rosa, G., Croxall, K., et al. 2014, ApJ, 796, 134

Dorodnitsyn, A., \& Kallman, T. 2012, ApJ, 761, 70

Elitzur, M. 2008, New Astron. Rev., 52, 274

Elitzur, M., Ho, L. C., \& Trump, J. R. 2014, MNRAS, 438, 3340

Fischer, T. C., Crenshaw, D. M., Kraemer, S. B., \& Schmitt, H. R. 2013, ApJS, 209, 1

Fischer, T. C., Crenshaw, D. M., Kraemer, S. B., Schmitt, H. R., \& Turner, T. J. 2014, ApJ, 785, 25

Gaskell, C. M., Goosmann, R. W., Merkulova, N. I., Shakhovskoy, N. M., \& Shoji, M. 2012, ApJ, 749, 148

Gezari, S., Hung, T., Cenko, S. B., et al. 2017, ApJ, 835, 144

Goodrich, R. W. 1989, ApJ, 340, 190

Goosmann, R. W., \& Gaskell, C. M. 2007, A\&A, 465, 129

Graham, M. J., Ross, N. P., Stern, D., et al. 2020, MNRAS, 491, 4925

Gravity Collaboration (Sturm, E., et al.) 2018, Nature, 563, 657

Grosset, L., Rouan, D., Gratadour, D., et al. 2018, A\&A, 612, A69

Husemann, B., Urrutia, T., Tremblay, G. R., et al. 2016, A\&A, 593, L9

Hutsemékers, D., Agís González, B., Sluse, D., Ramos Almeida, C., \& Acosta Pulido, J.-A. 2017, A\&A, 604, L3

Hutsemékers, D., Agís González, B., Marin, F., et al. 2019, A\&A, 625, A54

Kauffmann, G., Heckman, T. M., Tremonti, C., et al. 2003, MNRAS, 346, 1055

Kay, L. E. 1994, ApJ, 430, 196

Khachikian, E. Y., \& Weedman, D. W. 1971, ApJ, 164, L109

Kim, D.-C., Yoon, I., \& Evans, A. S. 2018, ApJ, 861, 51

Kinney, A. L., Schmitt, H. R., Clarke, C. J., et al. 2000, ApJ, 537, 152
Kishimoto, M., Kay, L. E., Antonucci, R., et al. 2002, ApJ, 565, 155

Konigl, A., \& Kartje, J. F. 1994, ApJ, 434, 446

Krolik, J. H., \& Begelman, M. C. 1988, ApJ, 329, 702

Krumpe, M., Husemann, B., Tremblay, G. R., et al. 2017, A\&A, 607, L9

LaMassa, S. M., Cales, S., Moran, E. C., et al. 2015, ApJ, 800, 144

Lovelace, R. V. E., Romanova, M. M., \& Biermann, P. L. 1998, A\&A, 338, 856

MacLeod, C. L., Ross, N. P., Lawrence, A., et al. 2016, MNRAS, 457, 389

MacLeod, C. L., Green, P. J., Anderson, S. F., et al. 2019, ApJ, 874, 8

Marin, F. 2016, MNRAS, 460, 3679

Marin, F. 2017, A\&A, 607, A40

Marin, F. 2018, A\&A, 615, A171

Marin, F., Goosmann, R. W., Gaskell, C. M., Porquet, D., \& Dovčiak, M. 2012, A\&A, 548, A121

Marin, F., Goosmann, R. W., \& Gaskell, C. M. 2015, A\&A, 577, A66

Martin, P. G., Thompson, I. B., Maza, J., \& Angel, J. R. P. 1983, ApJ, 266, 470

Mathis, J. S., Rumpl, W., \& Nordsieck, K. H. 1977, ApJ, 217, 425

McElroy, R. E., Husemann, B., Croom, S. M., et al. 2016, A\&A, 593, L8

Meisenheimer, K., Raban, D., Tristram, K., et al. 2008, The Messenger, 133, 36

Miller, J. S., \& Antonucci, R. R. J. 1983, ApJ, 271, L7

Nenkova, M., Sirocky, M. M., Ivezić, Ž., \& Elitzur, M. 2008a, ApJ, 685, 147

Nenkova, M., Sirocky, M. M., Nikutta, R., Ivezić, Ž., \& Elitzur, M. 2008b, ApJ, 685,160

Noda, H., \& Done, C. 2018, MNRAS, 480, 3898

Osterbrock, D. E. 1991, Rep. Prog. Phys., 54, 579

Penston, M. V., \& Perez, E. 1984, MNRAS, 211, 33P

Pier, E. A., \& Krolik, J. H. 1992, ApJ, 401, 99

Reeves, J. N., Porquet, D., Braito, V., et al. 2016, ApJ, 828, 98

Rojas Lobos, P. A., Goosmann, R. W., Marin, F., \& Savić, D. 2018, A\&A, 611, A39

Ruan, J. J., Anderson, S. F., Cales, S. L., et al. 2016, ApJ, 826, 188

Runnoe, J. C., Cales, S., Ruan, J. J., et al. 2016, MNRAS, 455, 1691

Sazonov, S., Churazov, E., \& Krivonos, R. 2015, MNRAS, 454, 1202

Shapovalova, A. I., Popović, L. Č., et al. 2019, MNRAS, 485, 4790

Shappee, B. J., Prieto, J. L., Grupe, D., et al. 2014, ApJ, 788, 48

Sheng, Z., Wang, T., Jiang, N., et al. 2017, ApJ, 846, L7

Simmons, J. F. L., \& Stewart, B. G. 1985, A\&A, 142, 100

Simpson, C., Wilson, A. S., Bower, G., et al. 1997, ApJ, 474, 121

Simpson, J. P., Colgan, S. W. J., Erickson, E. F., et al. 2002, ApJ, 574, 95

Stern, D., McKernan, B., Graham, M. J., et al. 2018, ApJ, 864, 27

Thompson, I. B., \& Martin, P. G. 1988, ApJ, 330, 121

Tran, H. D., Osterbrock, D. E., \& Martel, A. 1992, AJ, 104, 2072

Ulvestad, J. S., \& Wilson, A. S. 1984, ApJ, 285, 439

Walton, D. J., Nardini, E., Fabian, A. C., Gallo, L. C., \& Reis, R. C. 2013, MNRAS, 428, 2901

Wang, J., Xu, D. W., \& Wei, J. Y. 2018, ApJ, 858, 49

Wills, B. J., Wills, D., \& Breger, M. 2011, ApJS, 194, 19

Wu, X.-B., \& Han, J. L. 2001, ApJ, 561, L59

Yang, Q., Wu, X.-B., Fan, X., et al. 2018, ApJ, 862, 109

Young, S. 2000, MNRAS, 312, 567

Young, S., Corbett, E. A., Giannuzzo, M. E., et al. 1999, MNRAS, 303, 227

Zakamska, N. L., Schmidt, G. D., Smith, P. S., et al. 2005, AJ, 129, 1212

Zakamska, N. L., Strauss, M. A., Krolik, J. H., et al. 2006, AJ, 132, 1496 